\title{
LA ESTÉTICA DIGITAL EN EL CIBERESPACIO
}

\author{
AESTHETICS IN CYBERSPACE
}

\section{FABIÁN LEOTTEAU CASTRO}

Candidato a PhD en Diseño y Creación (Ciencias, Artes y Tecnología), Universidad de Caldas, magíster en Estudios del Caribe, Universidad Nacional de Colombia sede Caribe. Profesor de la Universidad del Atlántico (Colombia).

Artista plástico, gestor de proyectos, curador de arte, profesor investigador, director del grupo Investigaciones Visuales del Caribe (VIDENS), director de Anilla Cultural de Colombia y de la Comunidad de Arte y Cultura ARCU-RED, gestor de proyectos en e-art e investigador del ViveLab Atlántico. fabianleotteau@mail.uniatlantico.edu.co

\section{RESUMEN:}

Los artistas preocupados por la velocidad y el movimiento encuentran su propia dimensión en las nuevas formas de expresión, en las que pueden interactuar creativamente con ideas innovadoras aportándole a la revolución digital. De esta manera, el Ciberespacio es el escenario propicio para que el arte comparta sus propias coordenadas cibernéticas en el uso del nuevo soporte artístico para desarrollar proyectos colaborativos de contenidos estéticos digitales en las redes de alto rendimiento. Este artículo busca identificar y fomentar el uso de las nuevas herramientas tecnológicas para su aplicación en las prácticas artísticas en la comunidad ARCU-RED.

\section{PALABRAS CLAVE:}

cibercultura, arte y tecnología, revolución digital, comunidad ARCU-RED

\section{ABSTRACT:}

The artists concerned about the speed and movement, find their own dimension in the new forms of expression, which can interact creatively with innovative ideas giving it the digital revolution. Thus Cyberspace is the stage for you to share your own art Cyber coordinates using the new artistic support collaborative projects to develop digital aesthetic content in high-performance networks. This article is looking for identifying and promoting the use of new technological tools for application in artistic practices in community ARCU-RED.

\section{KEYWORDS:}

cyberculture, art and technology, digital revolution, community ARCU-RED. 


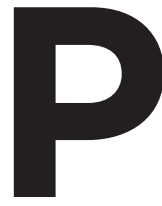
ara iniciar este debate comenzaremos hablando sobre el homo videns y la forma de adaptación al medio ambiente tratando de comprender el mundo, mediante el desarrollo de la inteligencia y la creatividad; en segunda instancia se hablará de cómo el arte aporta a las ciencias y a las humanidades hasta llegar a una revolución digital enfocada en el uso creativo de las nuevas tecnologías en las artes representativas y nuevos lenguajes multimediales; y por último se analizará el uso de las tecnologías avanzadas en las prácticas artísticas en la comunidad de Arte y Cultura ARCU-RED para concluir que el enriquecimiento interdisciplinario mediante el uso de las tecnologías avanzadas en el arte actual ofrece en el ciberespacio un proceso creativo, a partir del diseño y el intercambio de conocimientos estéticos, culturales, tecnológicos, científicos $\mathrm{y}$ visuales.

\section{LA VELOCIDAD Y EL MOVIMIENTO EN TIEMPO REAL}

El homo videns se adaptó al medio ambiente tratando de comprender el mundo, permitiéndole agudizar los sentidos a través de la creación de herramientas, las cuales ayudaron a desarrollar la inteligencia dándoles origen a los procesos de prácticas identitarias, apoyadas en las ciencias, la tecnología, el arte y las humanidades, representadas en los mundos simbólicos.

Por su parte, el concepto de arte ha cambiado en cada época de la historia, y en cada momento se ha retroalimentando creando nuevos significantes, hasta el punto de romper las fronteras de la cultura, constituida en la antropología visual, profundizando en las humanidades y en las ciencias, que si bien, de alguna manera, el mundo simbólico manifiesta sus costumbres, sus prácticas artísticas, su organización, sus aspiraciones e incluso sus contradicciones, también se da en la creación de objetos estético tangible e intangible. Hoy no sabemos con certeza hasta dónde llega el arte, solo sabemos que este se da desde hace mucho tiempo, es un concepto muy difícil de definir, pero ajustado a la realidad se involucra en procesos de prácticas y acciones del diario vivir que al ser señalado por Platón, al decir que el arte es la representación de las ideas formas, nos involucra en la esencia de la interpretación de las formas de la representación con unos valores estéticos intrínsecos.

Del mismo modo se puede decir que el artista en su laboratorio-taller ha experimentado en diversas técnicas, en diferentes épocas, las cuales influyeron en el modo de expresión y de comunicación; ello obedece a la búsqueda de nuevas fórmulas de representación, es decir, a la manera de crear nuevos mundos simbólicos. Uno de esos ejemplos es la prensa, que dio origen a la reproducción en serie de una imagen para llegar a más público. De esa imagen fija se pasó a la captura de la imagen haciendo un clip; esta a su vez, permitió que tuviera una secuencia, es decir, que de la imagen fija se pasara a la imagen en movimiento hasta llegar al cine. Otro ejemplo es la antropología visual como método de trabajo que influyó en contenidos y temas relacionados con la cultura en la que muchos artistas han encontrado sus nuevas formas de expresión.

A finales del siglo XIX y comienzos del XX la tendencia del puntillismo, creada por el neoimpresionista Georges Seurat, quien colocaba puntos de colores en vez de pinceladas sobre la tela, logró separar los tonos de colores por su posición, que al mirarlos a cierta distancia creaba en la retina combinaciones deseadas. Conocedor de las leyes físicas y fisiológicas, sus pinturas abrieron un campo de acción al conocimiento con los principios básicos de la óptica y del dibujo en la consolidación de la teoría de la imagen.

Estos y otros conceptos relacionados entre el arte y el desarrollo de las nuevas tecnologías incrementan las condiciones sociales, filosóficas y estéticas en diversos períodos, contribuyendo a la humanidad en cada época. Uno de esos aportes fue el estilo impresionista, cuando el artista preocupado por el fenómeno de la luz abrió las puertas a las ciencias, incursionando en el arte cinético o arte óptico basado en las formas esenciales de nuestra percepción del tiempo real y de la luz. Otro ejemplo es la obra de Marcel Duchamp Desnudo bajando la escalera, pintura que se inscribe en la exploración del cubismo como consecuencia del paso del tiempo, y exaltó el valor de lo coyuntural, lo fugaz y lo contemporáneo (Ortiz Herrera, 2008), como también la forma para identificar el movimiento del cuerpo humano y la dirección de la luz. Con esta pintura rompe en la cuarta dimensión identificada como el tiempo, descrita por el movimiento que se impregna en la mente y se convierte en arte retiniano, es decir, un arte netamente visual inimaginado en esos momentos. Asimismo, el proceso de industrialización abrió las puertas a la Revolución Industrial provocado por los descubrimientos científicos, los conflictos laborales, transformaciones demográficas, movimientos de masas y las tendencias del arte que influyeron en la sociedad en un período de constantes búsquedas artísticas.

Por otro lado, el documento "Fundación y manifiesto del futurismo de 1909", escrito por Filippo Tomassi Marinetti, plantea que "El amor al peligro, el valor, la audacia y la rebelión" (Marinetti, 1909), es reflejo de la sensibilidad donde la belleza, la máquina y la velocidad se consolidaban en las vanguardias futuristas, las que formulan el cambio del 'tempo' incrementando lo instantáneo propuesto por la Internet y la revolución digital, la cual está dando lugar al desarrollo de nuevos tipos de formato en el fortalecimiento de algunos movimientos artísticos de vanguardia como el futurismo y sus odas a la velocidad.

Asimismo, por su parte, al tratar de unir el arte y las ciencias como uno de los aportes que contribuyeron al proceso de planteamiento que hace Ortiz Herrera en su artículo "Informática y arte: arte + tecnología", en el que sostiene: Hacia 1919, apareció en Alemania una escuela de arte, arquitectura 
y diseño, conocida como Bauhaus; fue fundada por Walter Gropius (1883-1969) con dos propósitos: vincular el arte con los avances tecnológicos para que su aplicación fuera más útil a la sociedad y hacer que el artista tomara conciencia de su responsabilidad hacia la comunidad que le rodeaba y que reflejara esto en sus creaciones, dotándolas de una utilidad social evidente (Ortiz Herrera, 2008). Esto demuestra que el mundo del arte siempre ha estado a la vanguardia y le ha aportado muchos conceptos a las ciencias para proyectarse en modelos industriales.

Por consiguiente, los avances tecnológicos y el proceso de creación de objetos artísticos encuentran sus fundamentos en el pensamiento científico, filosófico, estético y matemático. Estas maneras de ver el arte generaron rupturas entre los géneros artísticos tradicionales, como la escultura y la pintura, con el fin de alcanzar un nuevo arte, experimentando con materiales no tradicionales, como la luz, el espacio, el tiempo y el pensamiento del espectador. En este sentido se puede decir que una de las contribuciones en la actualidad son las teorías estéticas soportadas en las tecnologías avanzadas, las cuales se conciben en la estructura de la creación artística como objeto estético reproducido digitalmente, permitiéndole acceder a mucho público y en simultánea.

Ahora bien, las ciencias sociales y las humanidades aportan un conocimiento profundo sobre la cultura, sobre una serie de métodos y procedimientos con proyección social. De hecho, en las humanidades es donde se fortalecen las comunidades.

Por ello se considera que la Internet de alta tecnología es un espacio que rompe las barreas, ya no hay editores, se filtra mucha equivocación, es decir, existe la liberta total; ahora hay mucha más creación que está saliendo a la luz. En este sentido, la gente que publica cosas en Internet busca contar historias en la red para beneficio propio, pero no todo lo que se publica pasa por un filtro de control de contenido, ni de calidad, y deja muchos vacíos a los internautas.

Asimismo, el público es cada vez más activo. Los modelos de desarrollo en tecnologías avanzadas suponen una nueva relación entre las personas y las máquinas, las cuales encuentran un proceso de readaptación. De igual manera el núcleo y motor de desarrollo en las ciudades se manifiesta a través de las nuevas tecnologías, en las que la cibercultura se sitúa con sus códigos visuales resignificados. En este sentido, los compromisos se desarrollan con métodos innovadores y participativos para identificar estrategias que fomenten la cultura.

Sin embargo, las prácticas artísticas apoyadas en el uso de las nuevas tecnologías contribuyen a la creatividad experimental con soportes innovadores y procesos continuos en la web 2.0, que al generar alternativas tecnológicas y educativas en el ciberespacio rompen las fronteras del arte y abren un campo de acción totalmente nuevo, que puede ser abordado desde el campo interdisciplinario a partir de los estudios visuales que posibilita sinergias entre universidades y centros culturales, donde la proyección de grupos de investigación en artes, permitan elevar las competencias en el ser, el hacer y el sentir.

Por lo que el análisis, diseño y ejecución de herramientas tecnológicas en espacios culturales y artísticos de la web 2.0 se ve expresado en estrategias de participación en el ámbito de las nuevas necesidades y realidades cultuales en redes sociales, donde asiste la mayor cantidad de público a través de la Internet.

Uno de los retos en los avances tecnológicos es la interacción del hombre con la máquina y la virtualidad, donde la imaginación no tiene límites, donde se confunde lo virtual con la realidad, tratando de dar respuestas al siglo XXI. De este modo encontramos algunos puntos de conexión de intercambio de contenidos y algunos otros dispositivos interactivos circunstanciales donde se pueden hacer exposiciones y eventos.

En estos momentos, con los adelantos tecnológicos en la última década, en que las artes han incursionado en nuevos espacios en la cultura digital, emplazada en una gran autopista estética, apoyada por instituciones oficiales y privadas através de proyectos artísticos, es cuando se pueden desarrollar propuestas colaborativas interdisciplinarias en la redes de alto rendimiento, como antecedentes a la revolución digital.

\section{REVOLUCIÓN DIGITAL}

Con el uso de las nuevas herramientas tecnologías se ha incrementado la experimentación en crear nuevos formatos estéticos, lo cual ha dado lugar a nuevos lenguajes que contribuyen a la creación de una revolución digital, en que la información y la comunicación requieren destrezas en su aplicación para interpretar las prácticas artísticas.

Es interesante cómo en algunos casos se plantea la urgencia de una nueva alfabetización sistematizada que responda a las necesidades derivadas de un nuevo orden social (Ortiz Herrera, 2008), con lo cual se reconoce que nos encontramos en la era de la informática y la simulación, en la que la creación en multimedia surge como principio básico de la alfabetización visual y en que los internautas abordan la significación que al expresarse libremente en la red, asumen un desafío personal e institucional al despertar gran interés en las nuevas generaciones.

Por consiguiente, la necesidad de repensar y reorientar los procesos ideológicos y las alternativas de políticas públicas en los medios de informática y las ciencias son determinantes a favor de una coherente lucha por la preservación y ampliación de la diversidad cultural, promoviendo esta, en torno a las redes sociales, espacios alternativos donde las herramientas tecnológicas abren las puertas a otras miradas hacia la aplicación de nuevo conocimiento científico; donde los seres humanos comienzan a modificar la naturaleza para satisfacer sus necesidades.

En este sentido, el concepto "tecnología" durante el siglo XX declaró una serie de aplicaciones en el conocimiento 
científico, tratando de comprender cómo las ingenierías le dan forma al mundo al abrir caminos complejos de procesos de investigación, donde los deseos y las necesidades le dan otra contemplación al mundo, promoviendo un desarrollo en las ciencias duras y en las humanidades.

Por su puesto que estos cambios generan conflictos y enfrentamientos, debido a que los riesgos que se toman no son claros y en muchos casos resultan muy confusos, por lo que es necesario el diseño de una alfabetización sobre tecnología digital apoyada en los lenguajes de multimedia, a fin de incorporar este sistema en un lenguaje común que despliegue el sentido de conceptos y palabras en tecnologías avanzadas, tan usadas últimamente, tales como: herramientas, dispositivos, Skype, la Internet, técnicas, organizaciones, software, sistemas, IP, MCU, en fin, todas sus combinaciones.

Cabe señalar que la revolución digital se inicia a partir de la relación entre la producción de estrategias militares y centros de producción de conocimientos, con sus antecedentes en las guerras mundiales, en las que se experimentaron algunas tecnologías, lo cual generó un caos geopolítico y ambiental planetario. Sin embargo, los inquietos "artistas" siguen experimentando.

Todo este caos geopolítico dio origen a lo que llamamos hoy Tecnología de la Información y Comunicaciones, las famosas TIC, que le apuntan a una nueva cultura en un espacio donde todos pueden acceder desde su ordenador, es decir que este sistema se desarrolla en la llamada cibercultura, fundamentada en el sistema de la globalización, en este sentido se recuerda que la revolución digital inició en los años 60 aproximadamente y finalizó cuando el sonido, la imagen y la palabra fueron separados y descifrados mediante el sistema binario.

A partir de lo anterior se planteó la equivalencia de archivos, convirtiéndolos en hipertexto en el mundo de la Web, es decir que actualmente estamos llenos de 0 y de 1 , lo que nos ha permitido pasar de un sistema analógico a uno digital en el que las imágenes, los sonidos y los datos se convierten en metadatos o metalenguajes, lo cual ha dado lugar a una interconexión globalizada con unos sistemas de flujos más amplios. Este proceso fue evolucionando poco a poco al punto las artes han creado y siguen creando otros tipos de experimentaciones una revolución digital. Un ejemplo claro se encuentra en el cine en el que la imaginación no tiene límites, la ilusión se hace posible, la mentira y la verdad juegan a la realidad, incrementando un estado de la mente, tal como lo sostiene Gonzalo Mayos cuando dice que en las sociedades avanzadas actuales cualquier hecho, "realidad" 0 "verdad" tiende a degradarse, ya sea a "espectáculo", ya sea a "consumo", ya sea -indistinguiblemente- a ambas cosas (Mayos Solana, 2006). Este planteamiento apuntan al concepto de "cultura y simulacro" de Baudrillard, al manifestar que la cultura se puede entender como "el conjunto de modos de vida y costumbres, conocimientos, grado de desarrollo artísticos, científico industrial, en una época o grupo social"; y simulacro como "imagen hecha a semejanza de una cosa o persona. Especie que forma la fantasía" (Gutiérrez Marín, 2003).

De este modo, la relación existente entre tecnología y sus usuarios construyen estructuras institucionales determinadas, tales como las que apuntó Walter Benjamín en su ensayo en la década del 30: "La obra de arte en la época de su reproductibilidad técnica" (Aguirre, 1978), cabe señalar que la inevitable transformación de las artes mediante diversos sistemas de operaciones tecnológicas participan de una relación sinérgica y dialéctica en la creación de simulacros en espacios inimaginados, es decir que mediante la reproducción técnica de la revolución digital el público puede elegir la oferta de contenidos con sus posibles aplicaciones.

Por lo anterior, el constante uso de la Internet facilita la libertad, lo interactivo entre la diversidad informativa y cultural de la comunicación social del usuario. Por consiguiente, las redes digitales con el uso de un metalenguaje, mediante la articulación de espectadores de la cultura mercantil se introducen en la simulación suplantando lo real por los signos de lo real, caso en el hecho lingüístico que entra a debatir con el lenguaje visual proporcionando un sentido ideológico de "lo bello", el cual entra en el mundo del arte y las nuevas tecnologías.

\section{LAS PRÁCTICAS ARTÍSTICAS Y EL USO DE LAS NUEVAS TECNOLOGÍAS}

Las artes y las ciencias siempre han estado articuladas, generando discursos visuales e introduciéndose y experimentando con soportes y técnicas totalmente diferentes en espacios creativos donde lo virtual es aprovechado por los avances tecnológicos; de algún modo, la ilusión y la imaginación en el arte son infinitas. Hoy en día es posible transfigurar nuevas formas de expresión y traspasar las fronteras del arte a través de los llamados: arte cibernético, arte telemático, arte digital, arte ciberpunk, entre otros; todos ellos se pueden ubicar en algún lugar en la cibercultura, término acuñado por Pierre Lévy al sostener que:

"El ciberespacio se construye como sistema de sistemas, pero, por ese mismo hecho, es también el sistema del caos. Encarnación máxima de la transparencia técnica, acoge, por su crecimiento sin contención, todas las opacidades del sentido. Diseña y rediseña varias veces la figura de un laberinto móvil, en expansión, sin plano posible, universal, un laberinto con el cual ni el mismo Dédalo habría soñado. Esa universalidad desprovista de significado central, ese sistema del desorden, esa transparencia laberíntica, la denomino 'universal sin totalidad'. Constituye la esencia paradójica de la cibercultura" (Schultz, 2006). 
Este ciberespacio produce la interacción de acontecimientos en la interface a maneras de interpretación del mundo virtualmente atrayente, creando una estética digital que permite que la velocidad y la simultaneidad se unan en el espacio/tiempo en las prácticas artísticas.

De tal manera que lo que antes era una ilusión visto muchas veces en el cine, ahora es una realidad gracias a la web 2.0, la cual posibilita el intercambio, la colaboración y la coproducción de contenidos estéticos entre artístas, investigadores, expertos en redes y académicos de diferentes lugares del mundo.

En este sentido, las películas Matrix, Yo Robot, Jurassic Park, la Momia, Ex-man, entre otras, han desarrollado una alfabetización audiovisual múltiple separando la imagen, el sonido y los metadatos. Este sistema, diseñado para el uso de lenguajes recientemente creados, contribuye al estudio de nuevas formas de comunicar, que resultan decisivas para la construcción de un mundo mejor, así sea en la virtualidad. Además, es de notar que el análisis crítico-reflexivo de la alfabetización digital, frente al puramente tecnológico, es el objetivo para transformar la información en conocimiento y hacer de este un elemento de colaboración y evolución digital en la sociedad; por lo que se necesita capacitar a sus integrantes en la alfabetización digital para vivir en un entorno flexible de aprendizajes, donde la verdadera alfabetización digital, como primer paso de una educación multimedia, responda a los fines últimos de la educación como herramienta de transformación social.

Por consiguiente, la comunidad de Arte y Cultura en Red ARCU-RED busca articularse con otras redes nacionales e internacionales para desarrollar proyectos colaborativos e interdisciplinarios y profundizar en la cultura visual, las cuales abren la mente a la imaginación en la gran autopista virtual. Imagínense ustedes como serán las nuevas maneras de ver el arte en alta definición, fenómeno que ya está ocurriendo. En este sentido, ARCU-RED Colombia se apoya en la cultura visual y en los estudios culturales con el propósito de indagar en las artes para construir desde la cultura nuevos discursos apoyados en la tecnología avanzada, para finalizar este apartado presento esta idea del profesor (Leotteau, 2009):

"En Estados Unidos los Estudios Visuales se transformaron en la Cultura Visual, proponiendo los espacios físicos y textuales entre lo popular y el mundo del arte, con un sentido crítico y de resistencia, tratando de legitimar los nuevos medios. Así aparecen la revista Después de la imagen en Rochester New York por iniciativa del Visual Estudies Workshop tratando de ajustarse a los nuevos conceptos de la Cultura visual, en la fotografía de uso cotidiano, el video y libros de artistas, desde

\begin{abstract}
un enfoque interdisciplinar planteando la posición del crítico en contra del historiador frente a la Cultura Visual. Estas metodologías están sujetas en las sociedades de masas sobreponiendo la historia del arte tradicional".
\end{abstract}

He ahí el gran reto.

\section{PROYECTOS DESARROLLADOS}

“Encuentro de MC" (Maestro de Ceremonias de hip-hop), desarrollado a través de la red RENATA. Para el desarrollo de esta actividad se diseñó una agenda de actividades con el fin de ajustar la infraestructura tecnológica, donde el hip-hop toma su iniciativa a partir de dos puntos de interconexión, uno desde el Museo de Antioquia de Medellín, con la participan de un DJ y el MC Canno, y desde la Universidad del Atlántico, en Barranquilla (Colombia), los "Mc" Doig-One y WM. En este sentido, una vez identificada las fallas y errores presentados ante el desplazamiento del audio por la distancia entre MedeLlín y Barranquilla, se diseñó un procedimiento que permitiría desarrollar la efectividad de la actividad programada. Con relación a la temática propuesta se planificó sacar un papel de una bolsa con un tema específico para improvisar acompañado de la música de hip-hop; en este caso la música vino desde el Museo de Antioquia, y en la Universidad del Atlántico cantaban a través de los micrófonos.

"Proyecto de Arte Visual Interactivo", en el que el público interviene haciendo cambios improvisados al desarrollo de la obra. La estética de causa-efecto hace referencia a la manera como los profesionales, artistas, ingenieros, técnicos, programadores instalan modos interactivos e intentan despertar la curiosidad del espectador mediante relaciones de tipo causa-efecto, cambiando el sentido por el que se enfocan la mirada y la tecnología que lo soporta. Esta práctica artística tuvo lugar en la Universidad del Atlántico de Barranquilla y la Universidad Tecnar de Cartagena con la participación de un grupo de egresados y estudiantes de ambas instituciones. La actividad se planificó a partir del análisis sitiogeografía de la ciudad de Cartagena de Indias y la relación de las problemáticas en la cibercultura, en común acuerdo con los artistas. En este sentido, el uso de software libre para hacer videos y la aplicación de herramientas se ajustan la infraestructura tecnológica de la actividad, teniendo en cuenta la preproducción de videos, en la que se adapta la música en vivo interconectada en la Red, desde cada ciudad.

"Cine Foro". Cada universidad perteneciente a la comunidad ARCU-RED, desarrolla su propio cineclub, el cual consiste en la programación de películas con una temática definida, respectando la Ley de Derecho de Autor y propiedad intelectual a través de la red de tecnología avanzada. En común acuerdo los líderes del cineclub programan el cine-foro y luego se 
publica en la web para que asistan desde las instituciones conectadas. Después de ver la película, los participantes debaten sobre el tema, los enfoques de la cámara, los planos, la calidad de la imagen, el color, la escenografía, entre otros temas relacionados con el contenido, y al final se expresan algunas las conclusiones sobre el tema tratado.

Por otro lado, se desarrolló un "Encuentro de Tambores", en el que se presentaron músicos y bailarines de música del carnaval de Barranquilla, desde las universidades Autónoma del Caribe y la Universidad del Atlántico, actividad que mezcla los ritmos musicales del carnaval, de manera que se hace un concierto en línea. En la Universidad del Atlántico hay un grupo denominado las Cantadoras del Río, producto de una investigación sobre el baile de tambora en el río Magdalena, el cual interactúa con el de la Universidad Autónoma del Caribe a través de los cueros del tambor, es decir, hay un diálogo de tambores en la Red de RENATA.

\section{CONCLUSIONES}

El entorno se ha transformado, no se reduce a lo que percibe nuestro ojo, se han multiplicado los escenarios y se interrelacionan en un espacio-tiempo imprevisible: el imaginario y lo "supuestamente real" se manifiestan en las pantallas $y$, a su vez, estas se encuentran por doquier, con diferentes tamaños, con movilidad, con posibilidades de generar una comunicación interactiva.

Para concluir se puede decir que el enriquecimiento interdisciplinario mediante el uso de las tecnologías avanzadas en el arte actual, ofrece en el ciberespacio un proceso creativo, a partir del diseño y el intercambio de conocimientos estéticos, culturales, tecnológicos, científicos y visuales a través de la red científica en RENATA, por lo que busca fomentar y exponer proyectos colaborativos e interdisciplinarios mediante videoconferencias, arte en línea, reflexiones estéticas, estudios visuales, productos de creación artística, productos de bienes y servicios, artísticos y culturales, encuentros de estudios culturales, entre otros, fundamentados en "la realidad virtual" en la Comunidad de Arte y Cultural en Colombia.

Asimismo, la ACC, como entidad de alta tecnología, comunica y divulga las prácticas identitarias culturales del territorio colombiano con proyección social en el orden nacional e internacional; por lo que el concepto de difusión cultural es retomado de Kroeber al decir que

La difusión es un proceso por el cual los elementos de los sistemas de cultura se diseminan. Obviamente está ligada a la tradición, en la medida en que la cultura material pasa de un grupo hacia otro. Sin embargo, como es usualmente entendida, la tradición se refiere a la transmisión de contenidos culturales, de una generación a otra (dentro del mismo grupo de población) y la difusión, de una población a otra. La tradición opera esencialmente en términos de tiempo, la difusión en términos de espacio (Gutiérrez Marín, 2003).
Es por ello que dentro de la comunidad ARCU-RED el diseño y desarrollo de proyectos en e-art generan nuevos conocimientos a partir de prácticas artísticas tradicionales, las cuales se convierten en procesos de reflexión en como el arte se encuentra en constantes cambios y experimentación.

Además, podríamos afirmar que ciertas expresiones, como el videoarte, o un arte relacionado con la tecnología, desarrollan una tendencia hacia el posicionamiento de actores colaboradores, de un género mixto y de una constante necesidad de experimentación de nuevos lenguajes expresivos e improvisados. El proceso de realización de una obra no termina con su exposición o presentación, ya que la nueva concepción colaborativa de arte conduce al espectador a ser parte activa de una obra en continua transformación.

\section{REFERENCIAS BIBLIOGRÁFICAS}

Aguirre, J. B. (1978). Discursos interrumpidos. La obra de arte en la época de su reproductividad técnica. . Madrid: Taurus .

Arce, C. F. (2004). Cultura y Somulacro de Jean Baudrillard. Revista Electrónica Glosa Didactica.

Gutiérrez Marín, M. (2003). Alfabetización digital. Algo más que ratones y teclas. Barcelona: Gedisa.

Leotteau, F. (2009). Una mirada al arte desde los estudios visuales. Congreso de Filosofía del Caribe (pág. 15). Barranquilla: Universidad del Atlántico.

Marinetti, F. T. (1909). Manifiesto Futurista. Recuperado el 25 de marzo de 2011, de http://elcraneo.8m.com/manifiestosfuturistas.htm

Mayos Solana, G. (2006). Baudrillard y la sociedad del simulacro. Recuperado el 18 de abril de 2011, de http://www.barcelonametropolis.cat/es/page. asp?id=21\&ui=363\#

Ortiz Herrera, A. (septiembre de 2008). Galería Informática. Recuperado el 25 de marzo de 2011, de http://www.inegi. gob.mx/inegi/contenidos/espanol/ciberhabitat/galeria/ informatica_y_arte/arte_mas_tecnologia.htm

Schultz, M. (2006). Filosofía y producciones digitales. Santiago de Chile: Colección TEORÍA 15.

\section{FORMA DE CITAR ESTE ARTÍCULO}

Leotteau Castro, Fabián (2013). La estética digital en el ciberespacio. Revista Arte y Diseño Facultad de Arquitectura, Arte y Diseño, Universidad Autónoma del Caribe, Barranquilla. ISSN 1692- 8555 Vol. 11 ( $\left.N^{\circ} .2\right)$. P.P 64-69 